\title{
TOPOLOGICAL MEASURE THEORY FOR DOUBLE CENTRALIZER ALGEBRAS
}

\author{
BY \\ ROBERT A. FONTENOT(1)
}

\begin{abstract}
The classes of tight, $\tau$-additive, and $\sigma$-additive linear functionals on the double centralizer algebra of a $C^{*}$-algebra $A$ are defined. The algebra $A$ is called measure compact if all three classes coincide. Several theorems relating the existence of certain types of approximate identities in $A$ to measure compactness of $A$ are proved. Next, permanence properties of measure compactness are studied. For example, the $C^{*}$-algebra tensor product of two measure compact $C^{*}$-algebras is measure compact. Next, the question of weak-star metrizability of the positive cone in the space of tight measures is considered. In the last part of the paper, another topology is defined and is used to study the relationship of measure compactness of $A$ and the property that the strict topology is the Mackey topology in the pairing of $M(A)$ with the tight functionals on $M(A)$. Also, in the last section of the paper is an extension of a result of Glickberg about finitely additive measures on pseudocompact topological spaces.
\end{abstract}

1. Introduction and preliminaries. The subject of topological measure theory was initiated in a now classical paper by Varadarajan [28]. This theory, having to do with measures on completely regular topological spaces, is nontrivial even when specialized to locally compact Hausdorff spaces. The theory of double centralizer algebras of $C^{*}$-algebras comes into the picture at this point, as it is a vehicle for extending the study of locally compact Hausdorff spaces to a noncommutative setting.

Briefly, the object of this paper is to extend to the setting of $C^{*}$-algebras a particular specialization of classical topological measure theory, viz., the case where all topological spaces considered are locally compact.

We first give a brief outline of topological measure theory. For more information, the reader is referred to [28, pp. 13-19]. Let $X$ denote a completely

Presented to the Society, January 16, 1974 under the title Measure compact $C^{*}$-alge. bras; received by the editors September 15, 1974.

AMS (MOS) subject classifications (1970). Primary 28A40; Secondary 46L05.

Key words and phrases. Tight, $\sigma$-additive, $\tau$-additive measure, double centralizer algebra.

(1) Part of the results in this paper are contained in the author's dissertation written under the direction of Professor H. S. Collins at Louisiana State University. The author wishes to thank Professor Collins for his encouragement and advice.

Copyright ¿ 1976, American Mathematical Society 
regular space and $C^{*}(X)$ denote the Banach algebra of all bounded complex-valued continuous functions on $X$. The Baire algebra of $X$, denoted $B_{a}^{*}(X)$, is the smallest algebra of subsets of $X$ generated by the zero-sets of all functions in $C^{*}(X)$. A positive Baire measure $m$ on $X$ is a finite, nonnegative real-valued, finitely-additive set function $m$ on $B_{a}^{*}(X)$ satisfying the condition $m(A)=\sup \{m(Z): Z$ $\subseteq A, Z$ a zero set of $X\}$ for all $A \in B_{a}^{*}(X)$. A Baire measure is any element of the complex linear span of the positive Baire measures. For any Baire measure $m$, let $|m|$ denote the total variation of $m$. The Banach space $M(X)$ of all Baire measures with norm $m \rightarrow|m|(X)$ is the adjoint of $C^{*}(X)$ in the natural way, via integration [28, Theorem 6].

At this point, let us introduce some notation to be used throughout this article. If $E$ is a topological vector space with a topology $T$ then by $E_{T}^{\prime}$ we mean the dual space of $E$ endowed with the topology $T$. If $E$ has a normed space structure, then by $E^{\prime}$ we mean the adjoint space of the normed space $E$. Note that $E$ may have one or more other topologies on it, but the notation $E^{\prime}$ refers specifically to the norm topology.

With this notation established, we now proceed to discuss the duality between $M(X)$ and $C^{*}(X)^{\prime}$. If $F \in C^{*}(X)^{\prime}$, then there is a unique Baire measure $m$ such that $\|F\|=\|m\|$ and $F(g)=\int g d m$ for all $g \in C^{*}(X)$. Conversely, if $m \in$ $M(X)$ and a functional $F$ is defined by the equation $F(g)=\int g d m$ for $g \in C^{*}(X)$, then $F \in C^{*}(X)^{\prime}$ and $\|F\|=\|m\|[28$, Theorem 6].

Three classes of Baire measures and functionals are of particular interest.

A Baire measure $m$ is said to be $\sigma$-additive if $m\left(Z_{n}\right) \rightarrow 0$ for every sequence $\left\{Z_{n}\right\}$ of zero-sets of $X$ such that $Z_{n+1} \subseteq Z_{n} \forall_{n}$ and $\cap Z_{n}=\varnothing$. A measure $m$ $\in M(X)$ is called $\tau$-additive if $m\left(Z_{p}\right) \rightarrow 0$ for every net $\left\{Z_{p}\right\}$ of zero-sets of $X$, such that $Z_{p} \subseteq Z_{q}$ if $p \geqslant q$, which satisfies $\cap Z_{p}=\varnothing$. Finally, a measure $m \in$ $M(X)$ is tight if, for every $\epsilon>0$, there is a compact set $K_{\epsilon} \subseteq X$ so that $|m|_{*}\left(X \backslash K_{\epsilon}\right)<\epsilon$ (where for $E \subseteq X,|m|_{*}(E)=\sup \{|m(Z)|: Z$ is a zero-set of $X, Z$ $\subseteq E\})$. Let $F \in C^{*}(X)^{\prime} . F$ is called $\sigma$-additive if $F\left(g_{n}\right) \rightarrow 0$ for every sequence $\left\{g_{n}\right\}_{n=1}^{\infty} \subseteq C^{*}(X)$ such that $g_{n+1} \leqslant g_{n}$ and $g_{n} \rightarrow 0$ pointwise on $X . F$ is called $\tau$-additive if $F\left(g_{p}\right) \rightarrow 0$ for every net $\left\{g_{p} \mid p \in P\right\} \subseteq C^{*}(X)$ such that $g_{p}$ $\leqslant g_{q}$ for $p \geqslant q$ and $g_{p} \rightarrow 0$ pointwise on $X$. Finally, $F$ is tight if $F\left(g_{p}\right) \rightarrow 0$ for every net $\left\{g_{p}\right\}$ such that $g_{p} \rightarrow 0$ uniformly on compact subsets of $X$. As might be expected, tight functionals, $\tau$-additive functionals, and $\sigma$-additive functionals correspond to tight measures, $\tau$-additive measures and $\sigma$-additive measures via the correspondence between $C^{*}(X)^{\prime}$ and $M(X)$ [28]. Under this identification, the classes of tight, $\tau$-additive and $\sigma$-additive functionals (measures) are denoted $M_{t}(X), M_{\tau}(X)$, and $M_{\sigma}(X)$, respectively. The central problem in topological measure theory is to determine when $M_{\tau}(X)=M_{\sigma}(X)$. Such spaces $X$ have been called measure compact or $B$-compact by various authors. 
The second concept needed in this article is that of the double centralizer algebra of a $C^{*}$-algebra $A$. The double centralizer of $A$, denoted $M(A)$, is the set of all pairs $(T, S)$ where $T$ and $S$ are maps from $A$ to $A$ satisfying $a T(b)=S(a) b$ for all $a, b \in A$. $M(A)$ has a $C^{*}$-algebra structure and contains $A$ as a two-sided ideal. The study of $M(A)$ for a $C^{*}$-algebra $A$ was initiated by R. C. Busby [3] as a tool for the study of extensions of $C^{*}$-algebras.

$M(A)$ contains an isometric isomorphic copy of $A$ imbedded as a two-sided ideal. The strict topology for $M(A)$, denoted $\beta$, is that topology generated by the seminorms $x \rightarrow\|x a\|$ and $x \rightarrow\|a x\|$ for $x \in M(A)$ and $a \in A$. The strict topology is a locally convex topology for $M(A)$ and $A$ is dense [3]. The strict topology is a generalization of the topology $\beta$ for $C^{*}(S)$ (S, a locally compact Hausdorff space) defined by R. C. Buck [2]. Let $M(A)_{\beta}$ denote $M(A)$ endowed with the strict topology. The double centralizer concept is of interest because it includes many important examples. For example, if $A$ is a commutative $C^{*}$-algebra, $A$ may be regarded as the algebra $C_{0}(S)(S=$ the maximal ideal space of $A)$ of continuous functions on $S$ vanishing at infinity. The double centralizer algebra of $C_{0}(S)$ has been shown to be $C^{*}(S)$ [3], [29]. Questions about $S$ may be studied by looking at the relationship between $C_{0}(S)$ and $C^{*}(S)$. As another example, consider the algebra of compact operators on the Hilbert space $H . M(A)$ is $B(H)$, the bounded linear operators on $H[3]$, [20] .

$M(A)$ has been studied extensively by D. C. Taylor [26], [27]. We shall use several of his results herein, including his characterization of the dual space $M(A)_{\beta}^{\prime}$. For further information on $M(A)$ refer to [3] , [26], [27].

As a last remark; note that the letter $S$ in this paper always denotes a locally compact Hausdorff space and $C_{0}(S)$ always denotes the continuous complexvalued functions on $S$ which vanish at infinity.

2. Basic results and permanence properties.

2.1 Definition. Let $A$ be a $C^{*}$-algebra. Suppose $\left\{f_{\alpha}\right\}$ is a net in $M(A)$. Write $f_{\alpha} \downarrow 0$ if the following three conditions are satisfied:

(1) $0 \leqslant f_{\alpha} \leqslant I$ for all $\alpha$;

(2) $f_{\alpha} \leqslant f_{\beta}$ for $\beta \leqslant \alpha$;

(3) $f_{\alpha} \rightarrow 0$ in the strict topology.

2.2 REMARK. If $A=C_{0}(S)$, the algebra of continuous functions on the locally compact Hausdorff space $S$ which vanish at infinity, we can replace (3) in 2.1 by the assumption that $f_{\alpha} \rightarrow 0$ pointwise on $S$, by Dini's theorem.

2.3 Definition. Let $F$ be a positive linear functional on $M(A)$. Then $F$ is said to be o-additive if for any sequence $\left\{f_{n}\right\} \subseteq M(A)$ such that $f_{n} \downarrow 0, F\left(f_{n}\right)$ $\rightarrow 0 . F$ is called $\tau$-additive if for any net $\left\{f_{\alpha}\right\} \subseteq M(A)$ such that $f_{\alpha} \downarrow 0, F\left(f_{\alpha}\right)$ $\rightarrow 0$. Finally, $F$ is called tight if $F$ is $\beta$ continuous. For any arbitrary linear 
functional $F \in M(A)^{\prime}$ write $F=F_{1}-F_{2}+i F_{3}-i F_{4}$, where $F_{1}, F_{2}, F_{3}$, and $F_{4}$ are positive linear functionals on $M(A) . F$ is said to be $\sigma$-additive ( $\tau$-additive, tight) if $F$ has a decomposition such that $F_{1}, F_{2}, F_{3}$, and $F_{4}$ are $\sigma$-additive ( $\tau$ additive, tight). Let $M_{\sigma}(A), M_{\tau}(A)$ and $M_{t}(A)$ denote the $\sigma$-additive, $\tau$-additive, and tight linear functionals in $M(A)^{\prime}$.

2.4 REMARK. Using the standard technique for extending positive functionals on an algebra to a Banach space of continuous functions (see $[6,2.6 .4]$ ) and the minimality property of the Lebesgue decomposition of measures, it is easy to see that $F$ in $M(A)^{\prime}$ is $\sigma$-additive ( $\tau$-additive, tight) iff the positive and negative parts of the Hermitian parts of $F$ have the same property.

2.5 Notation. For $F \in M(A)^{\prime}$ and $x \in M(A)$ let $F \cdot x$ and $x \cdot F$ denote the linear functionals on $M(A)$ defined by the equations $F \cdot x(y)=F(x y)$ and $x \cdot$ $F(y)=F(y x)$ for $y \in M(A)$. The functionals $F \cdot x$ and $x \cdot F$ are in $M(A)^{\prime}$ always and belong to $M(A)_{\beta}^{\prime}=M_{t}(A)$ if $x \in A[26,2.1-2.3]$.

2.6 Proposition. $M_{t}(A)=M_{\tau}(A) \subseteq M_{\sigma}(A)$.

Proof. All we need to prove is that if $F$ is a positive linear functional in $M_{\tau}(A)$, then $F \in M_{t}(A)$. Choose an increasing bounded approximate identity $\left\{e_{\lambda}\right\}$ for $A$ satisfying $0 \leqslant e_{\lambda} \leqslant I \forall \lambda$. Note that $I-e_{\lambda} \downarrow 0$ so that $F\left(I-e_{\lambda}\right)$ $\rightarrow 0$. Let $d_{\lambda}$ denote the positive square root of $I-e_{\lambda}$. Then for $x \in M(A)$

$$
\left|F \cdot d_{\lambda}(x)\right|^{2}=\left|F\left(d_{\lambda} x\right)\right|^{2} \leqslant F\left(I-e_{\lambda}\right) F\left(x^{*} x\right) \leqslant\|F\|\|x\|^{2} F\left(I-e_{\lambda}\right) .
$$

Thus

$$
\left\|F \cdot\left(I-e_{\lambda}\right)\right\| \leqslant\left\|d_{\lambda}\right\|\left\|F \cdot d_{\lambda}\right\| \leqslant\left\|d_{\lambda}\right\|\|F\|^{1 / 2} F\left(I-e_{\lambda}\right)^{1 / 2}
$$

Hence $\left\|F \cdot\left(I-e_{\lambda}\right)\right\| \rightarrow 0$ so $F \in M_{t}(A)[26]$.

In the classical case of topological measure theory [14], the support of a positive measure $\mu$ on a completely regular space is defined as the $\bigcap\{Z: Z \subseteq X$ is a zero set and $\mu(Z)=\mu(X)\}$. If the support of $\mu$ is the empty set, $\mu$ is said to be entirely without support [14]. The next two theorems develop the analogue of measures entirely without support.

2.7 Theorem [27, Proposition 2.5], [6, 2.11.7]. Let $f \in M(A)^{\prime}$. Then $f=f_{1}+f_{2}$ where $f_{1} \in M(A)_{\beta}^{\prime}$ and $f_{2} \in A^{\perp}=\left\{g \in M(A)^{\prime}: g \equiv 0\right.$ on $\left.A\right\}$. The decomposition is unique. Furthermore, if $f$ is a positive linear functional, then so are $f_{1}$ and $f_{2}$.

2.8 Definition. Let $A$ be a $C^{*}$-algebra. We say that $A$ is measure compact if $M_{\sigma}(A)=M_{t}(A)$. This definition coincides with that given in the commutative case, i.e., for the case $A=C_{0}(S)$. 
The next result shows that if $A$ is not measure compact, then the situation is the worst possible, i.e., there is an element of $M_{\sigma}(A)$ which annihilates $A$.

2.9 THEOREM. Suppose that $A$ is not measure compact. Then there is a nonzero positive linear functional in $M_{\sigma} \cap A^{\perp}$ (see 2.7).

Proof. By hypothesis, there is a positive linear functional $g$ in $M_{\sigma}(A)$ but not in $M_{t}(A)$. By 2.7, write $g=g_{1}+g_{2}$ with $g_{1}$ and $g_{2}$ positive linear functionals on $M(A)$ such that $g_{1} \in M(A)_{\beta}^{\prime}=M_{t}(A)$ and $g_{2} \in A^{\perp}$. Then $g_{2} \in M_{\sigma}(A)$ since $g_{2}=g-g_{1}$ and $M_{t}(A) \subseteq M_{\sigma}(A)$.

The next result is the analogue of the result that a Lindelöf ( $\sigma$ compact, in the locally compact case) topological space is measure compact.

2.10 THEOREM. Suppose $A$ has a countable approximate identity. Then $A$ is measure compact.

Proof. We may assume that $A$ has a bounded approximate identity $\left\{e_{n}\right\}$ satisfying $e_{n} \leqslant e_{m}$ for $n \leqslant m$ and $0 \leqslant e_{n} \leqslant I$ for all $n$. If $A$ is not measure compact, there is a nonzero positive linear functional $g$ in $M_{\sigma} \cap A^{\perp}$ by 2.9. Hence, $g\left(e_{n}\right)=0$ for all $n$. But $I-e_{n} \downarrow 0$ so $g\left(e_{n}\right) \rightarrow g(I)=\|g\| \neq 0$.

A set $X$ is said to have measure compact cardinal if the set $X$ with the discrete topology is measure compact, i.e., if each countably additive measure defined on all subsets of $X$ which vanishes on points of $X$ is identically zero. The next result is motivated by the result [13] that a paracompact space with measure compact cardinal is measure compact. See $[8$, p. 241] .

2.11 THEOREM. Suppose $A=\left(\Sigma A_{\lambda}\right)_{0}$ (see 3.1 for definition) where $\Lambda$ is a set having measure compact cardinal and each $A_{\lambda}$ is measure compact. Then $A$ is measure compact.

Proof. Note that $M(A)=\Sigma M\left(A_{\lambda}\right)$ [26, Lemma 3.1]. Suppose $A$ is not measure compact and that $g$ is a nonzero positive linear functional in $M_{\sigma}(A) \cap$ $A^{\perp}$. Note that $g \equiv 0$ on $M\left(A_{\lambda}\right)$ (i.e., the canonical image of $M\left(A_{\lambda}\right)$ in $M(A)$ ). Let $I_{\lambda}$ denote the identity in $M\left(A_{\lambda}\right)$. Let $b^{\lambda}$ denote that element of $M(A)$ whose $\lambda$ th coordinate is $I_{\lambda}$ and whose $\alpha$ th coordinate is zero, for $\alpha \neq \lambda$. For any subset $\Lambda_{0}$ of $\Lambda$, let $\mu\left(\Lambda_{0}\right)=g\left(\Sigma_{\lambda \in \Lambda_{0}} b^{\lambda}\right)$. Note that $\mu$ vanishes on points of $\Lambda$. A straightforward computation shows that $\mu$ is $\sigma$-additive as a measure on $\Lambda$. Also, $\mu(\Lambda)=g(I) \neq 0$. The existence of $\mu$ contradicts the assumption that $\Lambda$ has measure compact cardinal. Hence $A$ is measure compact.

2.12 CoROLlarY. Let $S$ be locally compact and paracompact with the discrete set $S$ measure compact. Then, $S$ is measure compact. 
Proof. See [8, p. 241] and use 2.10.

The concept of well-behaved approximate identity was introduced in [27]. A reasonable conjecture is that if $A$ has a well-behaved approximate identity, then $A$ is measure compact. We prove a special case of this theorem, for algebras having a series approximate identity [1] . A series approximate identity for a $C^{*}$. algebra is a family $\left\{f_{\alpha}\right\}_{\alpha \in \Gamma}$ of projections in $A$ such that, for each $a \in A$, $\left\|a\left(\Sigma_{\alpha \in F} f_{\alpha}\right) a-a\right\| \rightarrow 0$ as $F$ runs through the finite subsets of $\Gamma$. It is easy to see that the net $\left\{\Sigma_{\alpha \in F} f_{\alpha}: F\right.$ finite $\left.\subseteq \Gamma\right\}$ is a well-behaved approximate identity for $A$, where the $F$ 's are ordered by inclusion.

2.13 THEOREM Suppose $A$ has a series approximate identity $\left\{e_{\lambda}: \lambda \in \Lambda\right\}$ with $\Lambda$ having measure compact cardinal. Then $A$ is measure compact.

Proof. Assume $\Lambda$ is infinite. Let $F$ be a nonzero positive linear functional in $M_{\sigma}(A) \cap A^{\perp}$. For $\Lambda_{0} \subseteq \Lambda$, define $\mu\left(\Lambda_{0}\right)=F\left(\Sigma_{\lambda \in \Lambda_{0}} e_{\lambda}\right)$. Verify that $\mu$ is a nonzero $\sigma$-additive measure defined for all subsets of $\Lambda$, which vanishes on points of $\Lambda$.

2.14 Lemma [3]. Suppose $A$ and $B$ are $C^{*}$-algebras and $T: A \rightarrow B$ is $a$ surjective (onto) *-homomorphism. Then $\exists F: M(A) \rightarrow M(B)$ such that $F$ is a *homomorphism extending $T$ and such that $F$ maps the identity of $M(A)$ onto the identity of $M(B)$. Furthermore, $F$ is continuous when $M(A)$ and $M(B)$ are given their respective strict topologies.

2.15 Theorem. Suppose $T: A \rightarrow B$ is a surjective *-homomorphism and $A$ is measure compact. Then $B$ is measure compact.

Proof. Suppose $g$ is a positive linear functional in $M_{\sigma}(B) \cap B^{\perp}$. Let $F$ : $M(A) \rightarrow M(B)$ be the extension of $T$ whose existence is guaranteed by 2.14 . Let $F^{\prime}: M(B)^{\prime} \rightarrow M(A)^{\prime}$ denote the adjoint map. Let $h=F^{\prime}(g)$. If $a \in A, h(a)=$ $\left(F^{\prime}(g)\right)(a)=g(F(a))=g(T(a))=0$ since $g \equiv 0$ on $B$. Suppose $\left\{a_{n}\right\} \subseteq A$ and $a_{n} \downarrow 0$. Then $h\left(a_{n}\right)=g\left(F\left(a_{n}\right)\right) \rightarrow 0$ since $F$ preserves order, is norm decreasing and $\beta$-continuous. Thus $h \in M_{\sigma}(A)$ and $h \equiv 0$ on $A$. Since $A$ is measure compact, $h \equiv 0$. Let $I_{A}$ and $I_{B}$ denote the identity elements in $M(A)$ and $M(B)$ respectively. Then

$$
0=h\left(I_{A}\right)=\left(F^{\prime}(g)\right)\left(I_{A}\right)=g\left(F\left(I_{A}\right)\right)=g\left(I_{B}\right)=\|g\| .
$$

Hence, $g \equiv 0$, so $B$ is measure compact.

2.16 Corollary. If $A$ and $B$ are *-isomorphic $C^{*}$-algebras, then $A$ is measure compact if and only if $B$ is.

2.17 Corollary. Suppose $X$ and $Y$ are homeomorphic locally compact topological spaces. Then $X$ is measure compact iff $Y$ is. 
2.18 Corollary. Suppose $S$ is a measure compact topological space and $F$ a closed subset of $S$. Then $F$ is measure compact.

Proof. The restriction map $R: C_{0}(S) \rightarrow C_{0}(F)$ is surjective.

An important problem in topological measure theory is the determination of the Prohorov spaces. A completely regular space $X$ is called Prohorov if every weak-* compact subset of the positive tight measures on $X$ is uniformly tight [28]. It is known that locally compact spaces are Prohorov. Our next result is a generalization of this commutative result.

2.19 Theorem. Suppose $H \subseteq M(A)_{\beta}^{\prime}$ is $\beta$ weak-* compact and consists of positive linear functionals. Then $H$ is $\beta$-equicontinuous.

Proof. We use the criterion for $\beta$-equicontinuity in [26, Theorem 2.6]. Note that $H$ is norm bounded. Suppose that $\left\{e_{\lambda}: \lambda \in \Lambda\right\}$ is an approximate identity for $A$ such that $0 \leqslant e_{\lambda} \leqslant I \forall \lambda \in \Lambda$ and $\left\{e_{\lambda}\right\}$ is increasing. Let $\epsilon>0$. Since $I-e_{\lambda} \rightarrow 0$ in the strict topology, the sets $\left\{F \in H: F\left(I-e_{\lambda}\right)<\epsilon\right\}$ form a $\beta$-weak-* open cover of $H$. The compactness of $H$ implies that we can choose a finite set $\left\{\lambda_{i}\right\}_{i=1}^{n}$ from $\Lambda$ so that $H \subseteq \bigcup_{i=1}^{n}\left\{F \in H: F\left(I-e_{\lambda_{i}}\right)<\epsilon\right\}$. Choose $\lambda_{0}$ in $\Lambda$ such that $\lambda_{0} \geqslant \lambda_{i}, i=1, \ldots, n$. Then $\lambda \geqslant \lambda_{0}$ implies $0 \leqslant I-e_{\lambda} \leqslant I$ $-e_{\lambda_{i}}$ for $1 \leqslant i \leqslant n$ so that $F\left(I-e_{\lambda}\right)<\epsilon$ for all $F \in H$. Hence, letting $d_{\lambda}$ denote the positive square root of $I-e_{\lambda}$,

$$
\left\|d_{\lambda} \cdot F \cdot d_{\lambda}\right\|=d_{\lambda} \cdot F \cdot d_{\lambda}(I)=F\left(I-e_{\lambda}\right)<\epsilon
$$

for $\lambda \geqslant \lambda_{0}$. Thus

$$
\left\|\left(I-e_{\lambda}\right) \cdot F \cdot\left(I-e_{\lambda}\right)\right\|=\left\|d_{\lambda}^{2} \cdot F \cdot d_{\lambda}^{2}\right\| \leqslant\left\|d_{\lambda}\right\|^{2}\left\|d_{\lambda} \cdot F \cdot d_{\lambda}\right\|<\epsilon
$$

for $\lambda \geqslant \lambda_{0}$. Thus $H$ is $\beta$-equicontinuous by Taylor's criterion [26]

There are many questions suggested by our work on generalized topological measure theory. What we have presented here, together with the next result, is only a beginning. As a corollary to the theorem on tensor products below, we have that finite products of locally compact measure compact spaces are measure compact.

For this result, we need the concept of the $C^{*}$-tensor product of $C^{*}$-algebras. We sketch the construction and refer the reader to [22] for a more detailed treatment.

2.20 Definition. Let $A$ and $B$ be $C^{*}$-algebras and $A \otimes B$ denote the algebraic tensor product of $A$ and $B$, regarded as a set of bilinear forms on $A^{\prime} \times B^{\prime}$. Define involution for "elementary tensors" $a \otimes b$ by $(a \otimes b)^{*}=a^{*} \otimes b^{*}$ and extend linearly. If $(f, g) \in A^{\prime} \times B^{\prime}$, let $f \otimes g\left(\sum_{i=1}^{n} a_{i} \otimes b_{i}\right)=\sum_{i=1}^{n} f\left(a_{i}\right) g\left(b_{i}\right)$. If $f$ and $g$ are positive, $f \otimes g$ is positive [22]. The $C^{*}$-tensor product of $A$ and $B$ 
is the completion of $A \otimes B$ under the norm $\alpha_{0}$, defined for $x \in A \otimes B$ by $\alpha_{0}(x)$ $=\sup \left\{\left(f \otimes g\left(y^{*} x^{*} x y\right) / f \otimes g\left(y^{*} y\right)\right)^{1 / 2}: f\right.$ and $g$ are positive linear functionals of norm $\leqslant 1$ on $A$ and $B$, respectively, and $y \in A \otimes B$ such that $f \otimes g\left(y^{*} y\right) \neq 0$. From a slightly different viewpoint, if we let $\pi_{f \otimes g}$ denote the representation of $A \otimes B$ associated with the positive functional $f \otimes g\left[6\right.$, Chapter 2], then $\alpha_{0}(x)$ $=\sup \left\{\left\|\pi_{f \otimes g}(x)\right\|: f\right.$ and $g$ are positive linear functionals of norm $\leqslant 1$ on $A$ and $B$ respectively\}.

We shall let $A \otimes_{\alpha_{0}} B$ denote the $C^{*}$-tensor product of $A$ and $B$.

2.21 THEOREM. Suppose $A$ and $B$ are measure compact. Then so is $A \otimes_{\alpha_{0}} B$.

Proof. Regard $A$ as a subset of $M(A)$ and $B$ as a subset of $M(B)$ in the natural way. Suppose that $F$ is a $\sigma$-additive positive linear functional on $M\left(A \otimes_{\alpha_{0}} B\right)$ and $F \neq 0$. We shall show that $F \neq 0$ on $A \otimes B$. First, we show that $M\left(A \otimes_{\alpha_{0}} B\right)$ contains naturally imbedded copies of $M(A)$ and $M(B)$.

Let $a^{\prime} \in M(A)$ and $\Pi_{1}\left(a^{\prime}\right)=\left(L a^{\prime}, R a^{\prime}\right)$ where $L a^{\prime}$ is the linear map on $A \otimes$ $B$ defined by $L a^{\prime}\left(\Sigma a_{i} \otimes b_{i}\right)=\Sigma a^{\prime} a_{i} \otimes b_{i}$, for $\Sigma a_{i} \otimes b_{i} \in A \otimes B$, and $R a^{\prime}\left(\Sigma a_{i} \otimes b_{i}\right)=\Sigma a_{i} a^{\prime} \otimes b_{i}$. We verify that $R a^{\prime}$ and $L a^{\prime}$ are well defined. For if $\Sigma a_{i} \otimes b_{i} \equiv 0$, i.e., represents the zero form, so that $\Sigma \varphi\left(a_{i}\right) \psi\left(b_{i}\right)=0$ for all $\varphi$ $\in A^{\prime}, \psi \in B^{\prime}$, then $\Sigma a^{\prime} a_{i} \otimes b_{i} \equiv 0$ also, since if $\varphi \in A^{\prime}$ and $\psi \in B^{\prime}$, then $\Sigma \varphi\left(a^{\prime} a_{i}\right) \psi\left(b_{i}\right)=\Sigma p\left(a_{i}\right) \psi\left(b_{i}\right)=0$, where $p=\varphi \cdot a^{\prime}$ (see 2.5). Thus, $L a^{\prime}$ is well defined; similarly, $R a^{\prime}$ is well defined.

For boundedness, let us assume that $a^{\prime} \in A$. Let $\varphi$ be a positive linear functional on $B$. We want to show that

$$
\left\|\Pi_{\varphi \otimes \psi}\left(\sum a^{\prime} a_{i} \otimes b_{i}\right)\right\| \leqslant\left\|a^{\prime}\right\|\left\|\Pi_{\varphi \otimes \psi}\left(\sum a_{i} \otimes b_{i}\right)\right\| .
$$

Let $H_{\varphi}, H_{\psi}$, and $H_{\varphi \otimes \psi}$ be the Hilbert spaces constructed from $A, B$ and $A \otimes B$ by means of the positive functionals $\varphi, \psi$, and $\varphi \otimes \psi$. Sakai notes [22, p. 61] that $\Pi_{\varphi \otimes \psi}$ on $H_{\varphi \otimes \psi}$ is unitarily equivalent to $\Pi_{\varphi} \otimes \Pi_{\psi}$ on $H_{\varphi} \otimes H_{\psi}$ (where “ $\otimes$ " denotes the tensor product of Hilbert spaces defined in Dixmier [30, p. 21]. By making the appropriate identifications, we are able to restrict our attention to the pre-Hilbert space $A_{\varphi} \otimes B_{\psi}$ where $A_{\varphi}$ denotes the pre-Hilbert space constructed from $\varphi$ and $A$ and $B_{\psi}$ denotes the pre-Hilbert space constructed from $\psi$ and $B$. Let $I_{\psi}$ and $I_{\varphi}$ denote the identity operator on $H_{\psi}$ and $H_{\varphi}$ respectively. Then,

$$
\Pi_{\varphi} \otimes \Pi_{\psi}\left(\sum a^{\prime} a_{i} \otimes b_{i}\right)=\left(\Pi_{\varphi}\left(a^{\prime}\right) \otimes I_{\psi}\right) \circ\left(\Pi_{\varphi} \otimes \Pi_{\psi}\left(\sum a_{i} \otimes b_{i}\right)\right)
$$

Hence, 


$$
\begin{aligned}
\left\|\Pi_{\varphi \otimes \psi}\left(\sum a^{\prime} a_{i} \otimes b_{i}\right)\right\| & =\left\|\Pi_{\varphi} \otimes \Pi_{\psi}\left(\sum a^{\prime} a_{i} \otimes b_{i}\right)\right\| \\
& \leqslant\left\|\Pi_{\varphi}\left(a^{\prime}\right) \otimes I_{\psi}\right\|\left\|\Pi_{\varphi} \otimes \Pi_{\psi}\left(\sum a_{i} \otimes b_{i}\right)\right\| \\
& =\left\|\Pi_{\varphi}\left(a^{\prime}\right) \otimes I_{\psi}\right\|\left\|\Pi_{\varphi \otimes \psi}\left(\sum a_{i} \otimes b_{i}\right)\right\| .
\end{aligned}
$$

The first equality and the last follow because $\Pi_{\varphi \otimes \psi}$ and $\Pi_{\varphi} \otimes \Pi_{\psi}$ are unitarily equivalent, as noted above.

Our problem then reduces to computing the norm of the operator $\Pi_{\varphi}\left(a^{\prime}\right)$ $\otimes I_{\psi}$ on $A_{\varphi} \otimes B_{\psi}$. By using 2.22 (following this theorem) plus the fact that $\Pi_{\varphi}$ is norm decreasing, we see that

$$
\left\|\Pi_{\varphi}\left(a^{\prime}\right) \otimes I_{\psi}\right\| \leqslant\left\|\Pi_{\varphi}\left(a^{\prime}\right)\right\|\left\|I_{\psi}\right\| \leqslant\left\|a^{\prime}\right\| .
$$

Thus

$$
\left\|\Pi_{\varphi \otimes \psi}\left(\sum a^{\prime} a_{i} \otimes b_{i}\right)\right\| \leqslant\left\|a^{\prime}\right\|\left\|\Pi_{\varphi \otimes \psi}\left(\sum a_{i} \otimes b_{i}\right)\right\|
$$

so that $L_{a}$, is a bounded linear operator on $A \otimes B$ of norm $\leqslant\left\|a^{\prime}\right\|$ and so extends to $A \otimes_{\alpha_{0}} B$. Similarly, $R_{a}$, extends to all of $A \otimes_{\alpha_{0}} B$ with norm $\leqslant\left\|a^{\prime}\right\|$.

In the above discussion, we assumed that $a^{\prime} \in A$. Now suppose that $a^{\prime} \in$ $M(A)$ and that $\left\{a_{\alpha}^{\prime}\right\}$ is a net in $A$ such that $a_{\alpha}^{\prime} \rightarrow a^{\prime}$ in the strict topology. Choose $\left\{a_{\alpha}^{\prime}\right\}$ so that $\left\|a_{\alpha}^{\prime}\right\| \leqslant\left\|a^{\prime}\right\|$ for all $\alpha$. Then

$$
\begin{gathered}
\alpha_{0}\left(L_{a^{\prime}}\left(\sum a_{i} \otimes b_{i}\right)\right)=\alpha_{0}\left(\sum a^{\prime} a_{i} \otimes b_{i}\right) \\
=\sup \left\{\left(\frac{\varphi \otimes \psi\left(y^{*} \Sigma\left(a^{\prime} a_{i}\right) * \otimes b_{i}^{*}\left(\Sigma a^{\prime} a_{i} \otimes b_{i}\right) y\right)}{\varphi \otimes \psi\left(y^{*} y\right)}\right)^{1 / 2}:\right. \\
0 \leqslant \varphi \in A^{*},\|\varphi\| \leqslant 1,0 \leqslant \psi \in A^{*},\|\psi\| \leqslant 1, \\
\left.\varphi \otimes \psi\left(y^{*} y\right) \neq 0, y \in A \otimes B\right\}
\end{gathered}
$$

Suppose $y=\Sigma c_{j} \otimes d_{j}$. Since we may regard $\varphi$ as an element of $M(A)_{\beta}^{\prime}$, inserting $\Sigma c_{i} \otimes d_{i}$ for $y$ in the above expression and evaluating $\varphi \otimes \psi$, we get that 
$\alpha_{0}\left(\Sigma a^{\prime} a_{i} \otimes b_{i}\right)=\lim _{\alpha} \alpha_{0}\left(\Sigma a_{\alpha}^{\prime} a_{i} \otimes b_{i}\right)$. This last fact follows from the $\beta$-continuity of $\varphi$. Hence,

$$
\alpha_{0}\left(L_{\alpha^{\prime}}\left(\sum a_{i} \otimes b_{i}\right)\right) \leqslant \lim _{\alpha}\left\|a_{\alpha}^{\prime}\right\| \alpha_{0}\left(\sum a_{i} \otimes b_{i}\right) \leqslant\left\|\alpha^{\prime}\right\| \alpha_{0}\left(\sum a_{i} \otimes b_{i}\right) .
$$

Thus $\left\|L_{a^{\prime}}\right\| \leqslant\left\|a^{\prime}\right\|$ for arbitrary $a^{\prime} \in M(A)$ and a similar result holds for $R_{a^{\prime}}$. By using the fact that $\alpha_{0}$ is a cross norm $\left(\alpha_{0}(x \otimes y)=\|x\|\|y\|\right)$, we see that $\left\|R_{a^{\prime}}\right\|$ $=\left\|L_{a^{\prime}}\right\|=\left\|a^{\prime}\right\|$. It is also clear that if $x, y \in A \otimes B$, then $x L_{a^{\prime}}(y)=R_{a^{\prime}}(x) y$, so that by continuity of $L_{a^{\prime}}$ and $R_{a^{\prime}}$, we get that $\left(L_{a^{\prime}}, R_{a^{\prime}}\right) \in M\left(A \otimes_{\alpha_{0}} B\right)$.

We may summarize the preceding arguments by saying that $\Pi_{1}: M(A) \rightarrow$

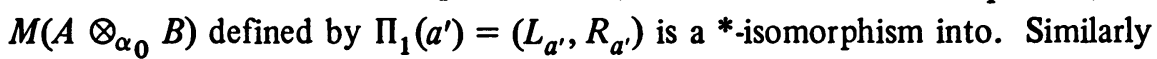
we may define a ${ }^{*}$-isomorphism $\Pi_{2}: M(B) \rightarrow M\left(A \otimes_{\alpha_{0}} B\right)$ with $\Pi_{2}\left(b^{\prime}\right)=$ $\left(L_{b^{\prime}}, R_{b^{\prime}}\right)$ for $b^{\prime} \in M(B)$ where $L_{b^{\prime}}\left(\Sigma a_{i} \otimes b_{i}\right)=\Sigma a_{i} \otimes b^{\prime} b_{i}$ and $R_{b^{\prime}}\left(\Sigma a_{i} \otimes b_{i}\right)=$ $\Sigma a_{i} \otimes b_{i} b^{\prime}$.

We are now ready to prove the theorem. First note the following fact: If $\left\{a_{n}^{\prime}\right\} \subseteq M(A)$ and $a_{n}^{\prime} \rightarrow 0_{\beta}$, then $\Pi_{1}\left(a_{n}^{\prime}\right) \rightarrow 0_{\beta}$, i.e., in the strict topology on $M\left(A \otimes_{\alpha_{0}} B\right)$ defined by $A \otimes_{\alpha_{0}} B$. For note that by the uniform boundedness theorem $\left\{\left\|a_{n}^{\prime}\right\|\right\}$ is bounded; thus it suffices to verify that $L_{a_{n}^{\prime}}$ and $R_{a_{n}^{\prime}}$ converge to zero pointwise as operators on $A \otimes B$. This is clear. Similarly if $\left\{b_{n}^{\prime}\right\} \subseteq M(B)$ and $b_{n}^{\prime} \rightarrow 0$ then $\Pi_{2}\left(b_{n}^{\prime}\right) \rightarrow 0$ in the strict topology of $M\left(A \otimes_{\alpha_{0}} B\right)$.

With these remarks in mind, one sees that the linear functional $F_{1}$ defined by the equation $F_{1}(x)=F\left(\Pi_{1}(x)\right)$, for $x \in M(A)$, is a nonzero $\sigma$-additive positive linear functional on $M(A)$. By measure compactness of $A, \exists a \in A^{+}$so that $F\left(\Pi_{1}\left(a^{2}\right)\right)>0$. Define a linear functional $F_{2}$ on $M(B)$ by the equation $F_{2}(y)=$ $F\left(\Pi_{1}(a) \Pi_{2}(y) \Pi_{1}(a)\right)$ and note that $F_{2}$ is nonzero, $\sigma$-additive, and positive. Measure compactness of $B$ then yields the existence of $b \in B^{+}$so that $F\left(\Pi_{1}(a) \Pi_{2}(b) \Pi_{1}(a)\right)>0$. Since it is easily checked that $\Pi_{1}(a) \Pi_{2}(b) \Pi_{1}(a)=a^{2}$ $\otimes b$, we have that $F\left(a^{2} \otimes b\right)>0$, i.e., $F$ does not annihilate $A \otimes B$. Since $F$ is an arbitrary $\sigma$-additive positive linear functional on $M\left(A \otimes_{\alpha_{0}} B\right)$, it follows that $A \otimes_{\alpha_{0}} B$ is measure compact.

We now state and prove the lemma used in this proof.

2.22 Lemma. Let $H_{1}$ and $H_{2}$ be two Hilbert spaces and $H_{1} \widetilde{\otimes} H_{2}$ be the completion of $H_{1} \otimes H_{2}$ with the inner product defined for elementary tensors by $\left\langle x_{1} \otimes x_{2}, y_{1} \otimes y_{2}\right\rangle=\left\langle x_{1}, y_{1}\right\rangle\left\langle x_{2}, y_{2}\right\rangle$ with $x_{1}, y_{1} \in H_{1}$ and $x_{2}, y_{2} \in H_{2}$. Suppose $T \in B\left(H_{1}\right)$ and $I_{2}$ is the identity operator on $H_{2}$. Let $T \otimes I_{2}$ be the operator on $H_{1} \otimes H_{2}$ defined by $T \otimes I_{2}\left(\Sigma x_{i} \otimes g_{i}\right)=\Sigma T\left(x_{i}\right) \otimes y_{i}$. Then $T \otimes$ $I_{2} \in B\left(H_{1} \otimes H_{2}\right)$ and $\left\|T \otimes I_{2}\right\| \leqslant\|T\|$ so that $T$ extends to all of $H_{1} \otimes H_{2}$.

Proof. Let $\Sigma x_{i} \otimes y_{i}$ be an element of $H_{1} \otimes H_{2}$. We may suppose that $\left\{y_{i}\right\}$ is an orthonormal set. Then 


$$
\begin{aligned}
& \left\|T \otimes I_{2}\left(\sum x_{i} \otimes y_{i}\right)\right\|^{2}=\left\|\sum T\left(x_{i}\right) \otimes y_{i}\right\|^{2}=\Sigma\left\|T\left(x_{i}\right)\right\|^{2} \\
& \leqslant\|T\|^{2} \Sigma\left\|x_{i}\right\|^{2}=\|T\|^{2}\left\|\Sigma x_{i} \otimes y_{i}\right\|^{2} \text {. }
\end{aligned}
$$

Hence $\left\|T \otimes I_{2}\right\| \leqslant\|T\|$.

3. Metrizability of $M_{t}(A)^{+}$. An interesting theorem due to Varadarajan [28, Theorem 13] states that $M_{\tau}(X)^{+}$(the positive cone in $\left.M_{\tau}(X)\right)$ is weak-* metrizable (in the relative weak-* topology from $\left.C_{b}(X)^{*}\right)$ iff $X$ is metrizable.

The specialization of the nontrivial half of the theorem to locally compact spaces states that if $S$ is locally compact and metrizable, then $M_{t}^{+}(S)$ is weak-* metrizable (recall that $M_{t}(S)=M_{\tau}(S)$ for locally compact $S$ ). In this section, we extend this result to a $C^{*}$-algebra setting. Our method, applied in the commutative case under the assumption that $S$ is locally compact, yields an easier proof of Varadarajan's result.

3.1 Definition. Suppose $\left\{X_{p} \mid p \in P\right\}$ is a collection of normed spaces (algebras). By the subdirect sum of the family $\left\{X_{p} \mid p \in P\right\}$, denoted $\left(\Sigma X_{p}\right)_{0}$, we mean $\left\{x=\left(x_{p}\right) \in \Pi X_{p}: \forall \epsilon>0, \exists F \subseteq P, F\right.$ finite, such that $\left\|x_{p}\right\|<\epsilon \forall p$ $\notin F\}$. Pointwise algebraic operations and norm $\|x\|=\sup \left\|x_{p}\right\|$ make $\left(\Sigma X_{p}\right)_{0}$ a normed space (algebra). By the $l^{\infty}$-sum of the family $\left\{X_{p} \mid p \in P\right\}$, denoted $\Sigma X_{p}$, we mean $\left\{x=\left(x_{p}\right) \in \Pi X_{p}\right.$ : sup $\left.\left\|x_{p}\right\|<\infty\right\}$. With pointwise operations and norm $\|x\|=\sup \left\|x_{p}\right\|, \Sigma X_{p}$ is a normed space (algebra). Finally, by the $l^{1}$-sum of the family of normed spaces $\left\{X_{p} \mid p \in P\right\}$, denoted $\left(\Sigma X_{p}\right)_{1}$, we mean $\{x=$ $\left.\left(x_{p}\right) \in \Pi X_{p}: \Sigma\left\|x_{p}\right\|<\infty\right\}$ where the $\Sigma\left\|x_{p}\right\|$ has the obvious meaning. Pointwise operations and norms given by $\|x\|=\Sigma\left\|x_{p}\right\|$ make $\left(\Sigma X_{p}\right)_{1}$ a normed space. Note that if all the $X_{p}$ are Banach spaces, so are $\left(\Sigma X_{p}\right)_{0}, \Sigma X_{p}$, and $\left(\Sigma X_{p}\right)_{1}$. Also, if all $X_{p}$ are $C^{*}$-algebras, so are $\left(\Sigma X_{p}\right)_{0}$ and $\Sigma X_{p}$.

We now state the main theorem of this section:

3.2 Theorem. Let $\left\{A_{p} \mid p \in P\right\}$ be a family of norm separable $C^{*}$-algebras and $A=\left(\Sigma A_{p}\right)_{0}$. Then the weak-* topology on $M_{t}^{+}(A)=\left(M(A)_{\beta}^{\prime}\right)^{+}$is metrizable.

3.3 Lemma. Let $A$ be a $C^{*}$-algebra, $F \in M_{t}(A)^{+}$, and $\left\{F_{p} \mid p \in P\right\}$ be a net contained in $M_{t}^{+}(A)$. Let I denote the identity of $M(A)$. Suppose $F_{p}(x) \rightarrow$ $F(x)$ for all $x \in A$ and $F_{p}(I) \rightarrow F(I)$. Then $F_{p} \rightarrow F$ weak-*.

Proof. We may assume that $\left\{F_{p}\right\}$ and $F$ are in the unit ball of $M_{t}(A)$ since $\left\|F_{p}\right\|=F_{p}(I) \rightarrow F(I)=\|F\|[6,2.1 .4]$. Let $x \in M(A),\|x\| \leqslant 1$, and $\epsilon>0$. Choose $a \in A$ such that $0 \leqslant a \leqslant I$ and $F(I-a)<\epsilon^{2} / 9$. Thus, also $F\left((I-a)^{2}\right)<\epsilon^{2} / 9$. Choose $p_{0}$ such that $p \geqslant p_{0}$ implies $F_{p}\left((I-a)^{2}\right)<\epsilon^{2} / 9$, 
and $\left|F_{p}(a x)-F(a x)\right|<\epsilon / 3$. Then $p \geqslant p_{0}$ implies that $\left|F_{p}(x)-F(x)\right|<\epsilon$. Thus $F_{p} \rightarrow F$ weak-*.

The next lemma is a special case of 3.2 that furnishes the idea for the proof of 3.2 .

3.4 Lemma. Let $A$ be a norm separable $C^{*}$-algebra. Then $M_{t}(A)^{+}$is weak-* metrizable.

Proof. Let $\left\{a_{n}\right\}_{n=1}^{\infty}$ be a countable dense set in the unit ball of $A$. Define a function $d$ on $M_{t}(A)^{+} \times M_{t}(A)^{+}$by

$$
d(F, G)=|F(I)-G(I)|+\sum_{n=1}^{\infty} \frac{1}{2^{n}}\left|F\left(a_{n}\right)-G\left(a_{n}\right)\right| .
$$

Clearly, $d$ is a metric on $M_{t}(A)^{+}$.

We now show that $d$ induces the weak-* topology. Suppose $\left\{F_{p} \mid p \in P\right\}$

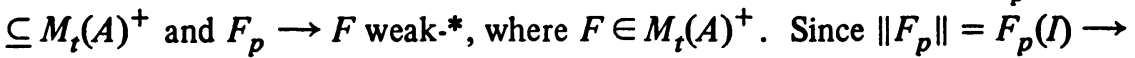
$F(I)=\|F\|$ and $d$ preserves nonnegative scalars $(d(c F, c G)=c d(F, G)$ for $c \geqslant 0)$ we may assume that $\left\|F_{p}\right\| \leqslant 1 \forall p \in P$ and $\|F\| \leqslant 1$. Let $\epsilon>0$ and choose an integer $N$ such that $\Sigma_{n=N+1}^{\infty} 1 / 2^{n}<\epsilon / 6$. Then choose $p_{0} \in P$ such that $p \geqslant p_{0}$ implies $\sup _{1 \leqslant n \leqslant N}\left|F_{p}\left(a_{n}\right)-F\left(a_{n}\right)\right|<\epsilon / 3$ and $\left|F_{p}(I)-F(I)\right|<\epsilon / 3$. Then $p \geqslant$ $p_{0} \Rightarrow d\left(F_{p}, F\right)<\epsilon$. Conversely, suppose that $d\left(F_{p}, F\right) \rightarrow 0$ for a net $\left\{F_{p}\right\} \subseteq$ $M_{t}(A)^{+}$and a functional $F$ in $M_{t}(A)^{+}$. We may assume that $\left\|F_{p}\right\| \leqslant 1 \forall p$ and $\|F\| \leqslant 1$. It is then easy to see that $F_{p}(a) \rightarrow F(a)$ for all $a \in A$. Since $F_{p}(I)$ $\rightarrow F(I)$ also, we have $F_{p} \rightarrow F$ weak-* by 3.3. This concludes the proof of 3.4 .

We now prove 3.2:

Proof. If $A=\left(\Sigma A_{p}\right)_{0}$, then $M(A)=\left(\Sigma M\left(A_{p}\right)\right)$ and $M_{t}(A)=\left(\Sigma M_{t}\left(A_{p}\right)\right)_{1}$ [26, Lemma 3.1].

For each $p \in P$, let $d_{p}$ be the metric on $M_{t}\left(A_{p}\right)^{+}$, constructed as in 3.4 such that $d_{p}$ induces the weak-* topology on $M_{t}\left(A_{p}\right)^{+}$. Recalling the construction, note that if $f$ and $g$ belong to $M_{t}\left(A_{p}\right)$, then $d_{p}(f, g) \leqslant 2\|f-g\|$.

Define a metric on $M_{t}(A)^{+}=\left(\Sigma M_{t}\left(A_{p}\right)\right)_{1}^{+}$by

$$
d(F, H)=|F(I)-H(I)|+\sum_{p \in P} d_{p}\left(F_{p}, H_{p}\right)
$$

where $F=\left(F_{p} \mid p \in P\right)$ and $H=\left(H_{p} \mid p \in P\right)$ belong to $M_{t}^{+}(A)$ and the sum has the obvious meaning. Note that the sum converges since

$$
\sum_{p \in P} d_{p}\left(F_{p}, H_{p}\right) \leqslant 2 \sum_{p \in P}\left\|F_{p}-H_{p}\right\|=2\|F-H\|<\infty .
$$

Also, $I$ in the above denotes $\left(I_{p}\right)$ where $I_{p}$ is the identity in $M\left(A_{p}\right)$. By straightforward computations and using 3.3, verify that $d$ induces the weak-* topology on $M_{t}(A)^{+}$. 
The last results in this section show that the fact that $M(A)$ has an identity and that we are considering the positive cone in $M_{t}(A)$ are vital to 3.2 .

3.5 REMARK. The following are equivalent:

(1) $M_{\sigma}(A)$ is a weak-* metrizable.

(2) $M_{t}(A)$ is a weak-* metrizable.

(3) $A$ is finite dimensional.

PROOF. We prove only the implication (2) $\Rightarrow(3)$ as the implications (1) $\Rightarrow$ (2) and (3) $\Rightarrow$ (1) are clear.

Suppose that $A$ is infinite dimensional. We shall first show that every weak-* neighborhood of zero in $M_{t}(A)$ contains an element of arbitrarily large norm. Let $V=\left\{F: F \in M_{t}(A)\right.$ and $\left|F\left(x_{i}\right)\right|<\epsilon$ for $1 \leqslant i \leqslant n$, where $\left.\left\{x_{i}\right\}_{i=1}^{n} \subseteq M(A)\right\}$ be a typical such neighborhood. Choose $a \in A, a \neq 0$. By using the Hahn-Banach theorem, construct a nonzero functional $F \in M(A)^{\prime}$ such that $F\left(a x_{i}\right)=0$ for $1 \leqslant$ $i \leqslant n$. Let $H=F \cdot a$ (see 2.5). Then $H \in M(A)_{\beta}^{\prime}[26]$ and $H\left(x_{i}\right)=0$ for $1 \leqslant i$ $\leqslant n$. Since any multiple of $H$ belongs to $V$, our claim is established.

Since $M_{t}(A)=M(A)_{\beta}^{\prime}$ is weak-* metrizable and each weak-* neighborhood of zero contains elements of arbitrarily large norm, we may choose a sequence $\left\{F_{n}\right\}_{n=1}^{\infty} \subseteq M_{t}(A)$ such that $\left\|F_{n}\right\| \geqslant n$ for each $n$ and $F_{n} \rightarrow 0$ weak-*. However, $\left\{F_{n}\right\}_{n=1}^{\infty} \subseteq M(A)^{\prime}$, the norm dual of $M(A)$ and must be norm bounded by the uniform boundedness theorem since $M(A)$ is a Banach space. This contradiction establishes 3.5 .

3.6 Remark. Suppose $A$ is a $C^{*}$-algebra without identity. Then $\left(A^{\prime}\right)^{+}$, the positive cone in the norm dual $A^{\prime}$, is weak-* metrizable iff $A$ is finite dimensional.

Proof. We may use the same idea as in 3.5 , provided we show that every weak-* zero neighborhood of zero in $A^{\prime}$ contains a positive element of arbitrarily large norm. It suffices to show that, given a finite set $\left\{x_{i}\right\}_{i=1}^{n} \subseteq A^{+}$, the positive cone of $A, \exists H \in\left(A^{\prime}\right)^{+}$such that $H\left(x_{i}\right)=0,1 \leqslant i \leqslant n$. Let $B$ be the $C^{*}$. algebra generated by $\left\{x_{i}\right\}, I$ the identity in $M(A)$, and $C=\{x+\lambda I: x \in B$ and $\lambda$ is a complex number be the $C^{*}$-algebra generated by $B$ and $I$. The functional $f$ on $C$ defined by the equation $f(x+\lambda I)=\lambda$ is positive and so extends to a positive functional $F$ on $M(A)$. Let $H$ be the restriction of $F$ to $A$.

4. The condition $M_{\sigma}(A)=M(A)^{\prime}$ and a conjecture on measure compactness. We begin this section by looking at a property equivalent to pseudocompactness of the spectrum in the commutative case [11].

4.1 Definition. A $C^{*}$-algebra is said to have Dini's property if every sequence $\left\{x_{n}\right\}_{n=1}^{\infty} \subseteq M(A)$ which satisfies $x_{n} \downarrow 0$ converges to zero in norm.

The next result generalizes an interesting result due to Glicksberg [11]. The idea of the proof is Varadarajan's [28].

4.2 Theorem. $A$ has Dini's property iff $M(A)^{\prime}=M_{\sigma}(A)$. 
Proof. Dini's property on $A$ implies that $M(A)^{\prime} \subseteq M_{\sigma}(A)$; since $M_{\sigma}(A) \subseteq$ $M(A)^{\prime}$ always holds (by definition), $M(A)^{\prime}=M_{\sigma}(A)$.

On the other hand, suppose that $\left\{a_{n}\right\}_{n=1}^{\infty} \subseteq M(A)$ such that $a_{n} \downarrow 0$ but $\left\|a_{n}\right\| \geqslant 1$ for all $n$. Let $h_{i}$ be a state (positive linear functional of norm one) of $M(A)$ such that $h_{i}\left(a_{i}\right)=\left\|a_{i}\right\|$, for $1 \leqslant i$. Note that for $i \geqslant 1$ and $p \geqslant i, h_{p}\left(a_{i}\right) \geqslant$ $h_{p}\left(a_{p}\right) \geqslant 1$.

Let $L$ be a Banach limit on $l^{\infty}[9$, p. 73] and define a functional $F$ on $M(A)$ by the equation $F(x)=L\left(\left\{h_{n}(x)\right\}\right)$. Clearly $F \in M(A)^{\prime}$ and $F$ is positive.

For any real sequence $\left\{s_{n}\right\}_{n=1}^{\infty} \subseteq l^{\infty}, \underline{\lim }\left\{s_{n}\right\} \leqslant L\left(\left\{s_{n}\right\}\right) \leqslant \varlimsup \lim \left\{s_{n}\right\}$ [9, p. 73]. Thus, $F\left(a_{i}\right)=L\left(\left\{h_{n}\left(a_{i}\right)\right\}\right) \geqslant \varliminf\left\{h_{n}\left(a_{i}\right)\right\}_{n=1}^{\infty} \geqslant 1$, since $h_{n}\left(a_{i}\right) \geqslant 1$ for $n \geqslant i$. Hence, $F \notin M_{\sigma}(A)$. This concludes the proof of 4.2 .

The next result we aim at states that if $\overline{M(A)}$ (the spectrum or structure space of $M(A)$; see $[6$, p. 60]) is Hausdorff, then the strict topology $\beta$ is the Mackey topology in the pairing $\left(M(A), M_{t}(A)\right)$ if $A$ is measure compact. This is a generalization of the specialization to locally compact spaces of a nice result due to Sentilles [24, Theorem 5.7]. Unfortunately, we do not get this result, as we need to prove that $A$ has one additional property; however, it is reasonable to believe that this property holds for all $C^{*}$-algebras and that proving this requires only a technical tool from the theory of inductive limits of topological vector spaces. Regardless, we do get an interesting result, 4.6 , as a by-product of our efforts.

We first define a topology $\beta_{1}$, on $M(A)$, in analogy to the definition of Sentilles and show that this topology is the Mackey topology in the pairing with its dual. We then show, under the assumption that $\widehat{M(A)}$ is Hausdorff, that $M(A)_{\beta_{1}}^{\prime}=M_{\sigma}(A)$.

4.3 Definition. A two-sided ideal $J \subseteq M(A)$ is called admissible if $J$ is a $C^{*}$-subalgebra with a countable approximate identity such that $A \subseteq J$. For each admissible ideal $J$, let $\beta_{J}$ be the locally convex topology on $M(A)$ defined by the seminorms $x \rightarrow\|a x\|$ and $x \rightarrow\|x a\|$ for $x \in M(A)$ and $a \in J$. Define the topology $\beta_{1}$ on $M(A)$ as the intersection of the topologies $\beta_{J}$ or, equivalently, as the inductive limit of the topologies $\beta_{J}$.

4.4 REMARK. It follows from $[26,2.5]$ that the sets $V=\{x \in M(A)$ : $\|a x\| \leqslant 1$ and $\|x a\| \leqslant 1\}$ for $a \in J^{+}$is a base at zero for the topology $\beta_{J}$. Note also that $\beta \leqslant \beta_{1}$ since $\beta \leqslant \beta_{J}$ for each admissible ideal $J$ since each such $J \supseteq A$.

4.5 Proposition. (a) $M(A)_{\beta_{1}}^{\prime}=\bigcap\left\{M(A)_{\beta_{J}}^{\prime}: J\right.$ is an admissible ideal of $M(A)\}$.

(b') $H \subseteq M(A)_{\beta_{1}}^{\prime}$ is $\beta_{1}$-equicontinuous iff $H$ is $\beta_{J}$-equicontinuous for each admissible ideal $\mathrm{J}$.

(c) The Mackey topology in the pairing $\left(M(A), M(A)_{\beta_{1}}^{\prime}\right)$ is $\beta_{1}$.

Proof. Parts (a) and (b) follow from the definition of $\beta_{1}$ as the inductive limit of the topologies $\beta_{J}$. 
For part (c), let $F$ be a weak-* compact set in $M(A)_{\beta_{1}}^{\prime} \subseteq M(A)^{\prime}$. From [27, Theorem 2.1] we have that $M(J)=M(A)$ for each admissible ideal $J$. Results of Taylor [27, Theorem 3.1 and Corollary 3.4] imply that every weak-* compact subset of $M(J)_{\beta}^{\prime}=M(A)_{\beta_{J}}^{\prime}$ is $\beta_{J}$-equicontinuous, since $J$ has a countable approximate identity. Thus $F$ is $\beta_{J}$-equicontinuous for each $J$, and hence, $F$ is $\beta_{1}$ equicontinuous by 4.5 (b). Thus $\beta_{1}$ is the Mackey topology as claimed [21, p. 62].

4.6 THEOREM. Assume that $\widehat{M(A)}$, the structure space of $M(A)[6, p .60]$, is Hausdorff. Then $M(A)_{\beta_{1}}^{\prime}=M_{\sigma}(A)$.

Proof. Suppose $F$ is a positive linear functional in $M_{\sigma}(A)$. To show $F$ belongs to $M(A)_{\beta_{1}}^{\prime}$, it suffices to show $F$ is in $M(A)_{\beta_{J}}^{\prime}$ for each admissible ideal $J$. Let $J$ be an admissible ideal and $\left\{e_{n}\right\}_{n=1}^{\infty}$ a countable increasing bounded approximate identity in $J$ such that $0 \leqslant e_{n} \leqslant I$ where $I$ denotes the identity in $M(A)$. Then $I-e_{n} \downarrow 0$ so $F\left(I-e_{n}\right) \rightarrow 0$. Let $c_{n}=\left(I-e_{n}\right)^{1 / 2}$. Thus $\left\|c_{n} \cdot F \cdot c_{n}\right\|$ $\rightarrow 0$ (see 2.5 and $[6,2.1 .9])$. Hence,

$$
\left\|\left(I-e_{n}\right) \cdot F \cdot\left(I-e_{n}\right)\right\| \leqslant\left\|c_{n}\right\|^{2}\left\|c_{n} \cdot F \cdot c_{n}\right\| \rightarrow 0,
$$

so $F$ is $\beta_{J}$-continuous $[26,2.1],[25]$.

For the converse, let $\epsilon>0,\left\{x_{n}\right\}_{n=1}^{\infty} \subseteq M(A)$, and $x_{n} \downarrow 0$. Considering the continuous functions $\pi \rightarrow\left\|\pi\left(x_{n}\right)\right\|$ on $\widehat{M(A)}$, let $K=\bigcap_{n=1}^{\infty}\{\pi \in \widehat{M(A)}$ : $\left.\left\|\pi\left(x_{n}\right)\right\| \geqslant \epsilon / 2\right\}$. Note that $K$ is compact.

Let $I_{K}=\{x \in M(A): \pi(x)=0 \forall \pi \in K\}$. We will show that $I_{K}$ is an admissible ideal of $M(A)$.

First note that $\hat{A}$, the structure space of $A$, is a subset of $M(A)$ since $A$ is an ideal of $A$, i.e., $\hat{A}$ may be identified with an open subset of $\widehat{M(A)},\{\pi \in \widehat{M(A)}$ : $\pi$ is not identically zero on $A$ \} [6]. We will show that $K \cap \hat{A}$ is void. Let $\pi_{0} \in$ $\hat{A}$ and choose, by virtue of Urysohn's lemma, a continuous function $h$ such that $h\left(\pi_{0}\right)=1$ and $h=0$ on $\widehat{M(A)} \backslash \hat{A}$. By means of the Dauns-Hofmann Theorem [5], [7] applied to the algebra $M(A)$, let $z \in M(A)$ such that $\pi(z)=h(\pi) \pi(I)$ for all $\pi \in \widehat{M(A)}$ (here $I$ denotes the identity in $M(A)$ ). Note that $z \in A$ since $\pi(z)$ $=0$ for all $\pi \in \widehat{M(A)} \backslash \hat{A}$ and $\widehat{M(A)} \backslash \hat{A}$ may be canonically identified with the structure space of the (quotient) algebra $M(A) / A[6]$. We now show that $\pi_{0} \notin$ $K$. Since $x_{n} \rightarrow 0_{\beta},\left\|z x_{n}\right\| \rightarrow 0$ so

$$
\begin{aligned}
\left\|\pi_{0}\left(x_{n}\right)\right\| & =\left\|\pi_{0}\left(I x_{n}\right)\right\|=\left\|\pi_{0}(I) \pi_{0}\left(x_{n}\right)\right\|=\left\|\left[h\left(\pi_{0}\right) \pi_{0}(I)\right] \pi_{0}\left(x_{n}\right)\right\| \\
& =\left\|\pi_{0}(z) \pi_{0}\left(x_{n}\right)\right\|=\left\|\pi_{0}\left(z x_{n}\right)\right\| \leqslant\left\|z x_{n}\right\| \rightarrow 0 .
\end{aligned}
$$

Therefore $\pi_{0} \notin K$, hence $\hat{A} \cap K$ is void as claimed. With this established, we see that $A \subseteq I_{K}$.

We next demonstrate that $I_{K}$ has a countable bounded approximate iden- 
tity. Let $B=\{f: f$ is a continuous complex function on $\widehat{M(A)}$ and $f \equiv 0$ on $K\}$. Since $K$ is a compact $G_{\delta}$ in $\widehat{M(A)}, B$ has a countable approximate identity consisting of continuous functions $\left\{g_{n}\right\}_{n=1}^{\infty}$ satisfying $0 \leqslant g_{n} \leqslant 1$ for all $n$. Using the Dauns-Hofmann Theorem applied to $M(A)$ [5], [7], choose a sequence $\left\{z_{n}\right\}_{n=1}^{\infty}$ of Hermitian elements in $M(A)$ such that $\pi\left(z_{n}\right)=g_{n}(\pi) \pi(I)$ for all $\pi \in \widehat{M(A)}$. Observe that $\left\{z_{n}\right\} \subseteq I_{K}$. It is straightforward to verify that $\left\{z_{n}\right\}$ is an approximate identity for $I_{K}$.

We continue with the proof that $M(A)_{\beta_{1}}^{\prime} \subseteq M_{\sigma}(A)$. Let $F \in M(A)_{\beta_{1}}^{\prime}$ with $\|F\| \leqslant 1$. Then $F$ is $\beta_{I_{K}}$-continuous, so by $[26,2.1]$ and results in [25] we may factor $F=H \cdot a$ where $a \in I_{K}$ such that $\|a\| \leqslant 1$ and $\|H\| \leqslant 2$ (see 2.5). Let $\epsilon$ $>0$ be as previously chosen and $0=\{\pi \in M(A):\|\pi(a)\|<\epsilon / 2\}$. Note $K \subseteq 0$ since $a \in I_{K}$. Then $\exists N$ such that $n \geqslant N \Rightarrow\left\{\pi:\left\|\pi\left(x_{n}\right)\right\| \geqslant \epsilon / 2\right\} \subseteq 0$. Hence

$$
\begin{aligned}
n & \geqslant N \Rightarrow\left\|F\left(x_{n}\right)\right\|=\left\|H\left(a x_{n}\right)\right\| \leqslant 2\left\|a x_{n}\right\|=2 \sup _{\pi}\left\|\pi\left(a x_{n}\right)\right\| \\
& \leqslant 2 \sup _{\pi}\|\pi(a)\|\left\|\pi\left(x_{n}\right)\right\|<\epsilon .
\end{aligned}
$$

Thus $F\left(x_{n}\right) \rightarrow 0$ and hence $F \in M_{\sigma}(A)$ since $\left\{x_{n}\right\}$ was an arbitrary sequence in $M(A)$ such that $x_{n} \downarrow 0$.

This concludes the proof of 4.6.

Conjecture. Let $A$ be a measure compact $C^{*}$-algebra such that $M(A)$ has a Hausdorff structure space. Then $\beta=\beta_{1}$; hence $\beta$ is the Mackey topology in the pairing $\left(M(A), M_{t}(A)\right)$.

4.7 Proposition. Let $\left\{x_{p} \mid p \in P\right\}$ be a net in $M(A)$ such that $0 \leqslant x_{p} \leqslant I$ for all $p \in P$. Then $x_{p} \rightarrow 0 \beta$ iff $x_{p} \rightarrow 0$ uniformly on weak-* compact sets of positive tight measures.

Proof. Let $S^{+}$denote positive functionals in the unit sphere of the norm dual $M(A)^{\prime}$. For each $a \in A^{+}, a \cdot S^{+} \cdot a$ (see 2.5) is a weak-* compact set of positive tight measures. Thus $\sup _{f \in S}\left\|f\left(a x_{p} a\right)\right\| \rightarrow_{p} 0$; hence $\left\|a x_{p}^{1 / 2}\right\|=\left\|a x_{p} a\right\|^{1 / 2}$ $\rightarrow 0$. So

$$
\left\|x_{p} a\right\|=\left\|a x_{p}\right\| \leqslant\left\|a x_{p}^{1 / 2}\right\|\left\|x_{p}^{1 / 2}\right\| \rightarrow 0 .
$$

Thus $x_{p} \rightarrow 0 \beta$. The converse is clear since weak-* compact sets of positive measures are equicontinuous by 2.19 .

4.8 EXAMPLE. The result in 4.7 comes about as close as one can come to generalizing the commutative result which states that $\beta$ is the topology of uniform convergence on the weak-* compact sets of positive tight measures [24, Theorem 5.5].

Let $T$ denote the locally convex topology on $M(A)$ with seminorms $x \rightarrow$ $\|a x a\|$ for $x \in M(A)$ and $a \in A^{+}$. It is easy to see that $T \leqslant \beta$ and that $M(A)_{T}^{\prime}=$ $M(A)_{\beta}^{\prime}=M_{t}(A)$. It is also fairly easy to see that $T$ agrees with the topology of 
uniform convergence on weak-* compact sets of $M_{t}(A)$ on bounded sets of $M(A)$. Let $A$ denote the compact operators on an infinite-dimensional Hilbert space $H$; $M(A)$ is then $B(H)$, the algebra of bounded linear operators on $H$. Consider the restriction of $T$ and $\beta$ to the Hermitian part $S_{h}$ of the unit ball in $B(H)$. It is easily seen that $T$ coincides with the weak operator topology on $S_{h}$ and $\beta$ coincides with the strong operator topology on $S_{h}$. It is well known that the weak and strong operator topologies do not coincide on $S_{h}$; thus $\beta$ and the uniform convergence topology are distinct.

4.9 Question. Is 4.7 true when $\beta$ is replaced by $\beta_{1}$ ? In the commutative case $\beta_{1}$ is shown to be the topology of uniform convergence on weak-* compact subsets of $M_{\sigma}(A)^{+}$; this result is deduced using the analogous result for $\beta$ and the fact that $\beta$ is locally solid [23]. Unfortunately $\beta$ is not in general locally solid; e.g., if $A$ is the algebra of compact operators on an infinite-dimensional Hilbert space $H, \beta$ on $M(A)=B(H)$, the bounded linear operators on $H$, is not locally solid. Nevertheless, the author believes that 4.7 holds for $\beta_{1}$ also and that only technicalities involved in the definition of the inductive limit topology stand in the way of a proof. The commutative result is used to prove that $X$ measure compact $\Rightarrow \beta=\beta_{1}$, hence $\beta$ is the Mackey topology in the pairing $\left(C^{*}(X), M_{t}(A)\right)$. We seek a noncommutative extension of this result.

4.10 REMARK. If the answer to 4.9 is yes, then the following result obtains: A measure compact and $M(A)$ has a Hausdorff structure space $\Rightarrow \beta=\beta_{1}$ and $\beta$ is the Mackey topology in the pairing $\left(M(A), M_{t}(A)\right)$.

We give a proof of this claim. Since $\beta \leqslant \beta_{1}$ and $\beta$ is the finest locally convex topology agreeing with itself on bounded sets of $M(A)[26,2.7]$ it suffices to show that for a bounded net $\left\{f_{p}\right\} \subseteq M(A), f_{p} \rightarrow 0 \beta \Rightarrow f_{p} \rightarrow 0 \beta_{1}$. By decomposing each $f_{p}$ into its Hermitian parts and then using polar decomposition on each part we may assume that $0 \leqslant f_{p} \leqslant I$ for each $p \in P$. Since $f_{p} \rightarrow 0 \beta$, $f_{p} \rightarrow 0$ uniformly on weak-* compact sets of $M_{t}^{+}(A)=M_{\sigma}^{+}(A)$; our assumption that 4.8 holds for $\beta_{1} \Rightarrow f_{p} \rightarrow 0 \beta_{1}$. Thus $\beta=\beta_{1}$ as claimed.

We have seen the importance of Question 4.9 in proving the result mentioned in 4.10. Hopefully, a way of answering 4.9 in the affirmative can be found.

\section{BIBLIOGRAPHY}

1. C. A. Akemann, Interpolation in $W^{*}$-algebras, Duke Math. J. 35 (1968), 525-533. MR 37 \#4626.

2. R. C. Buck, Bounded continuous functions on a locally compact space, Michigan Math. J. 5 (1958), 95-104. MR 21 \#4350.

3. R. C. Busby, Double centralizers and extensions of $C^{*}$-algebras, Trans. Amer. Math. Soc. 132 (1968), 79-99. MR 37 \#770.

4. J. B. Conway, The strict topology and compactness in the space of measures, Trans. Amer. Math. Soc. 126 (1967), 474-486. MR 34 \#6503.

5. J. Dauns and K. H. Hofmann, Representation of rings by sections, Mem. Amer. Math. Soc. No. 83 (1968). MR 40 \#52. 
6. J. Dixmier, Les $C^{*}$-algèbres et leur représentations, 2 ième éd., Cahiers Scientifiques, fasc. 29, Gauthier-Villars, Paris, 1969. MR 39 \#7442.

7. - The ideal center of a $C^{*}$-algebra, Duke Math. J. 35 (1968), 375-382. MR 37 \#5703.

8. J. Dugundji, Topology, Allyn and Bacon, Boston, Mass., 1965. MR 33 \#1824.

9. N. Dunford and J. T. Schwartz, Linear operators. I: General theory, Pure and

Appl. Math., vol. 7, Interscience, New York, 1958. MR 22 \#8302.

10. D. H. Fremlin, D. J. H. Garling and R. Haydon, Bounded measures on topological spaces, Proc. London Math. Soc. (3) 25 (1972), 115-136. MR 49 \#9144.

11. I. Glicksberg, The representation of functionals by integrals, Duke Math. J. 19 (1952), 253-261. MR 14, 288.

12. E. Hewitt, The ranges of certain convolution operators, Math. Scand. 15 (1964), 147-155. MR $32 \# 4471$.

13. R. B. Kirk, Locally compact, B-compact spaces, Nederl. Akad. Wetensch. Proc. Ser. A 72 = Indag. Math. 31 (1969), 333-344. MR 41 \#201.

14. Measures in topological spaces and B-compactness, Nederl. Akad. Wetensch. Proc. Ser. A 72 = Indag. Math. 31 (1969), 172-183. MR 39 \#7410.

15. J. D. Knowles, Measures on topological spaces, Proc. London Math. Soc. (3) 17 (1967), 139-156. MR 34 \#4441.

16. W. Moran, The additivity of measures on completely regular spaces, J. London Math. Soc. 43 (1968), 633-639. MR 37 \#225.

17. Measures and mappings on topological spaces, Proc. London Math. Soc. 19 (1969), 493-508. $507-524$.

18. - Measures on metacompact spaces, Proc. London Math. Soc. 20 (1970),

19. S. Mosiman and R. F. Wheeler, The strict topology in a completely regular setting: relations to toplogical measure theory, Canad. J. Math. 24 (1972), 873-890. MR 48 \#6909.

20. G. Reid, A generalization of $W^{*}$-algebras, Pacific J. Math. 15 (1965), 1019-1026. MR 33 \#4701.

21. A. P. Robertson and W. J. Robertson, Topological spaces, Cambridge Univ. Press, New York, 1964. MR 28 \#5318.

22. S. Sakai, $C^{*}$-algebras and $W^{*}$-algebras, Springer-Verlag, New York, 1970.

23. H. Schaeffer, Topological vector spaces, Macmillan, New York, 1966. MR 33 \#1689.

24. F. D. Sentilles, Bounded continuous functions on a completely regular space, Trans. Amer. Math. Soc. 168 (1972), 311-336. MR 45 \#4133.

25. F. D. Sentilles and D. C. Taylor, Factorization in Banach algebras and the general strict topology, Trans. Amer. Math. Soc. 142 (1969), 141-152. MR 40 \#703.

26. D. C. Taylor, The strict topology for double centralizer algebras, Trans. Amer.

Math. Soc. 150 (1970), 633-643. MR 44 \#7302.

27. - A general Phillips theorem for $C^{*}$-algebras and some applications. Pacific J. Math. 40 (1972), 477-488. MR 46 \#7913.

28. V. S. Varadarajan, Measures on topological spaces, Mat. Sb. 55 (97) (1961), $35-$ 100; English transl., Amer. Math. Soc. Transl. (2) 48 (1965), 161-228. MR 26 \#6342.

29. J. Wang, Multipliers of commutative Banach algebras, Pacific J. Math. 11 (1961), 1131-1149. MR 25 \#1462.

30. J. Dixmier, Les algèbres d'opérateurs dans l'espace Hilbertien, 2nd ed., GauthierVillars, Paris, 1969.

\section{DEPARTMENT OF MATHEMATICS, OAKLAND UNIVERSITY, ROCHESTER, MICHIGAN 48063}

Current address: Department of Mathematics, Whitman College, Walla Walla, Washington 99362 